\title{
AUTOMATIC NAVIGATION SYSTEM WITH MULTIPLE SENSORS
}

\author{
Xiaojun Wan, Gang Liu* \\ Key Laboratory of Modern Precision Agriculture System Integration Research, China \\ Agricultural University, P.O. Box 125, Qinghuadonglu 17, Haidian District, Beijing, \\ 100083, P. R. China \\ * Corresponding author, Address: P.O. Box 125, China Agricultural University, Qinghua \\ Donglu 17, Haidian District, Beijing, 100083, P. R. China, Tel: +86-10-62736741, Fax: +86- \\ 10-62736746,Email: pac@cau.edu.cn
}

\begin{abstract}
An automatic navigation system with multiple sensors including a vision sensor, RTK GPS and an electronic-compass was developed. Data from the sensors were fused by a feedback algorithm. And the steering angle was calculated based on the fuzzy logic module. A steering controller was developed to control steering using guidance information obtained from the sensors. A golf-car was taken as the research platform which was equipped with an angle sensor, an accelerometer and a steering controller. The row-crop was selected as the interested crop. Navigation of the vehicle in the farmland could be realized by the system.
\end{abstract}

Keywords: GPS, machine vision, navigation, sensor fusion, PID, steering controller

\section{INTRODUCTION}

Over the past centuries, many changes have occurred on agricultural field production machinery. With the increasing working speed and vehicle size, an operator has to devote more energy to operate the vehicle (Wilson, 2000).

Wan, X. and Liu, G., 2008, in IFIP International Federation for Information Processing, Volume 259; Computer and Computing Technologies in Agriculture, Vol. 2; Daoliang Li; (Boston: Springer), pp. 769-776. 
Fatigue impedes the operator to operate the vehicle, especially during the tight constraints of harvest. The operator becomes one of the greatest limitations to the increasing vehicle performance. Therefore, developing automatic navigation system for agricultural vehicles is essential.

Automatic navigation systems attempt to relieve the operator from many, if not all, of the tasks involved in navigating agricultural vehicles. Automatic navigation has potential to reduce operator fatigue and improve positioning accuracy of the vehicle (Gerrish et al., 1997). A number of agricultural navigation techniques have been developed or demonstrated in recent years, such as machine vision, GPS, inertial positioning system, radar, ultrosonic, etc. But generally, a single sensor system can supply only parts of the environment information. The precision of the navigation is sometimes unbelievable. Therefore the navigation system with sensor fusing has become more and more popular in recent years. And the combination of machine vision and GPS becomes most popular (Wilson, 2000).

Automatic navigation system should be able to detect posture of the vehicle, calculate proper steering angle, and steer the vehicle according to the angle. The posture is the position and orientation of the vehicle (Kanayama and Hartman, 1989). Different automatic navigation systems have been developed for agricultural vehicles during the past several decades. Although each system uses different techniques to navigate the vehicle, most of the systems generally need the same navigation parameters, including heading angle and offset, to control steering. Heading angle is the angle between the vehicle centerline and the desired path, and offset is the displacement of the vehicle central mass off the desired path. More precise navigation parameters could be obtained by fusing the data acquired from different sensors. The fuzzy logic module was used to calculate a steering angle. Finally, a steering controller was installed on the vehicle to steer the vehicle following the desired path automatically.

This paper presented a solution to challenging problems for automatically navigated agricultural vehicles with multiple sensors. This solution included obtaining navigation parameters with CCD, GPS and electronic-compass, fusing multi-sensor data for real-time vehicle navigation, calculating the steering angle and steering the vehicle following the desired path with a steering controller. A feedforward-plus-PID steering control algorithm was used to compensate the variation in the vehicle dynamics for ensuring a satisfactory steering performance. A golf-car installed with an angle sensor, an accelerometer and a steering controller was taken as the research platform.

The configuration of the automatic navigation system was shown in Fig. 1. 


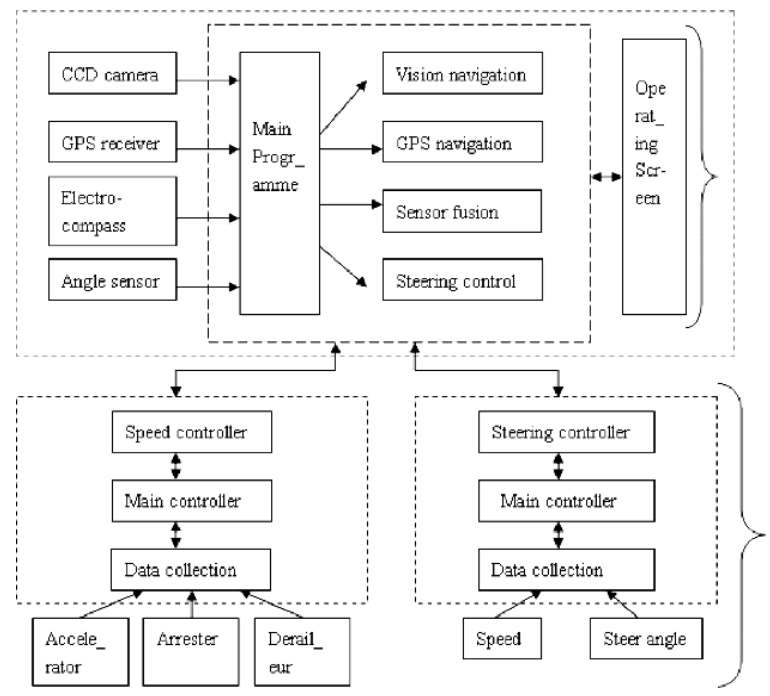

Fig. 1. The configuration of the automatic navigation system

\section{MULTIPLE SENSORS AND SENSOR FUSION}

\subsection{Obtaining navigation parameters based on machine vision}

Machine vision can be used to navigate agricultural vehicles automatically when the row structure is distinguishable in the farmland. It has the technological characteristics closely resembling those possessed by a human operator, and thus has great potential for implementation of the vehicle navigation system (Wilson, 2000). The flowchart to obtain the navigation directrix was stepped in Fig. 2.

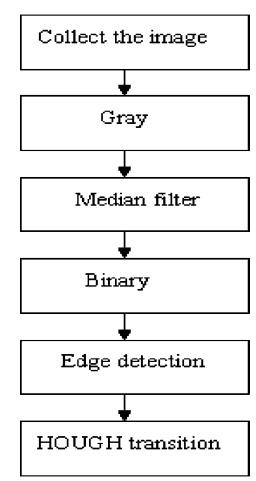

Fig. 2. The flowchart to obtain navigation directrix 
The first step of the algorithm was to collect the images from the farmland by the CCD camera. The second step was to convert the color image into gray by $2 \mathrm{G}-\mathrm{R}-\mathrm{B}$. The third step was to filter the image to reduce the noise. The forth step was to convert the image into a binary one by selecting the threshold automatically with Dajin method. The fifth step was edge detection to detect the boundary between the crop and the non-crop. Ultimately, the Hough transition was used to obtain parameters of the navigation directrix for the guidance line could be treated as a straight line.

Once the directrix was acquired, the following was the calculation of the vehicle projection coordinate in the image coordination. Camera calibration was shown in Fig. 3.

\begin{tabular}{|c|c|c|c|}
\hline ay & 0 & version & - \\
\hline Ime & Read & Extract grid & Calibrati \\
\hline Show Extrin & Reproject on images & Analyse & Recomp com \\
\hline Add/Sup & $\mathrm{Sz}$ & L & Exit \\
\hline Comp & ndistortim & Export calib data & callib \\
\hline
\end{tabular}

Fig. 3. The calibration toolbox of the camera

The projection relationship between the world coordination and the image coordination could be found.

$$
s\left[\begin{array}{c}
u \\
v \\
1
\end{array}\right]=M_{1} \times M_{2} \times\left[\begin{array}{c}
X_{W} \\
Y_{W} \\
Z_{W} \\
1
\end{array}\right]
$$

where $\left[\begin{array}{ll}u & v\end{array}\right]^{T}$ is the coordinate in the image coordination.

$\left[\begin{array}{lll}X_{W} & Y_{W} & Z_{W}\end{array}\right]^{T}$ is the coordinate in the world coordination.

$M_{1}$ is the internal parameters matrix of the camera.

$M_{2}$ is the external parameters matrix of the camera.

The vehicle coordinate in the world coordination is $(0,0,0)$, using the projection relationship formula, the vehicle coordinate in the image coordination can be calculated. Then the offset and heading angle are obtained.

$$
\begin{aligned}
& d=\frac{\left|A x_{0}+B y_{0}+C\right|}{\sqrt{A^{2}+B^{2}}} \\
& \theta=a \tan \frac{\left(x_{2}-x_{1}\right)}{\left(y_{2}-y_{1}\right)}
\end{aligned}
$$

where $\left(x_{0}, y_{0}\right)$ is the vehicle coordinate in the image coordination. 
$A, B, C$ are the beeline equation coefficient of the directrix.

$\left(x_{1}, y_{1}\right)$ is the begin point coordinate of the directrix.

$\left(x_{2}, y_{2}\right)$ is the end point coordinate of the directrix.

\subsection{Obtaining navigation parameters based on GPS}

GPS-based navigation technique can be used in many farmland operations such as tillage, planting, cultivating, harvesting, etc. It has the potential to achieve completely autonomic navigation. The flowchart to obtain navigation parameters by GPS was shown in Fig. 4 .

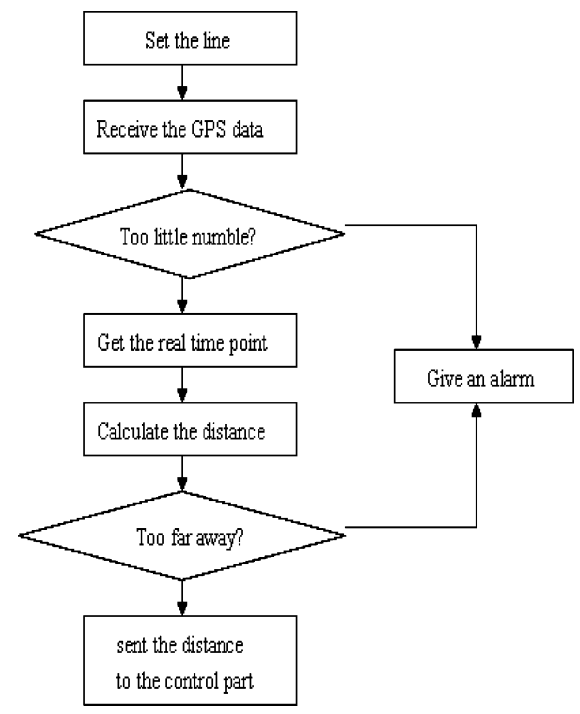

Fig. 4. The flowchart to obtain navigation parameters

Firstly, to set the end point and the begin point of the row. Based on the method that two points determine one line, the row line was got. GPS was connected to the computer through RS-232, received the associated data from satellites and abstracted the longitude and latitude from the data. By coordination transition, $\mathrm{X}$ and $\mathrm{Y}$ coordinates of the vehicle in the 54coordination were got. Once the real time position of the vehicle was got, the distance between the row and the vehicle could be calculated. An alarm signal was set when the vehicle going far away from the row, and the alarm distance could be set manually.

To integrate the sensors into a sensor fusion module required unifying coordinate systems of all the sensors. The GPS provided position information of the vehicle referring to the global coordination. The machine vision provided information in the image coordination. To make it compatible to the directrix information obtained from the vision sensor, it was necessary to convert the global coordinates to the image coordinates as 
well. However, it was necessary to correct the error caused by the vehicle inclination before mapping it to the vehicle coordination.

$$
\left[\begin{array}{l}
x_{c} \\
y_{c} \\
z_{c}
\end{array}\right]=\left[\begin{array}{l}
x_{a} \\
y_{a} \\
z_{a}
\end{array}\right]+\left[\begin{array}{lll}
a_{11} & a_{12} & a_{13} \\
a_{21} & a_{22} & a_{23} \\
a_{31} & a_{32} & a_{33}
\end{array}\right]\left[\begin{array}{l}
a \\
b \\
h
\end{array}\right]
$$

When the variation in the farmland elevation was limited, the 2-D GPS coordinates could be converted into the image coordinates.

$$
\left[\begin{array}{l}
x^{g}{ }_{v} \\
y^{g}{ }_{v}
\end{array}\right]=\left[\begin{array}{ll}
b_{11} & b_{12} \\
b_{21} & b_{22}
\end{array}\right]\left[\begin{array}{l}
x_{c} \\
y_{c}
\end{array}\right]
$$

\subsection{Sensor fusion}

The three sensors can navigate the vehicle separately or together. The block diagram of multiple navigation sensor fusion module was shown in Fig. 5.

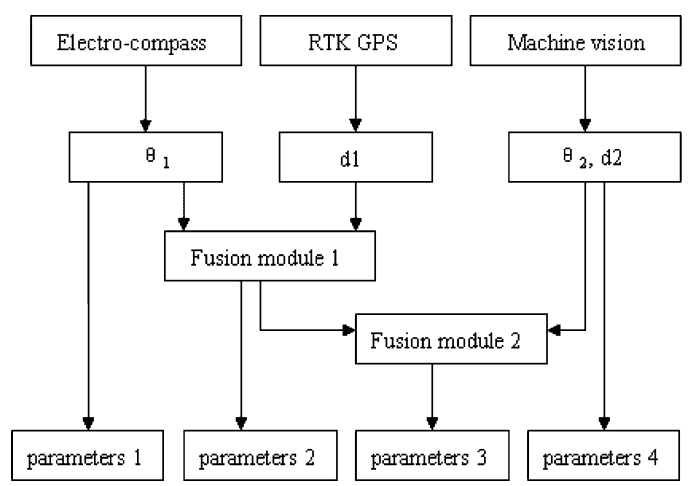

Fig. 5. The block diagram of multiple navigation sensor fuision module

The sensor fusion module evaluated the information acquired from different sensors and selected the chief navigation sensor using a rule-based method. With a corresponding fusion algorithm, more appropriate heading angle and offset information could be obtained in real-time.

Fusion algorithm based on feedback was presented in fusion module 2 and the precision still needed to be validated. The following was the fusion formula, which was mainly based on the fuzzy logic fusion.

$$
\begin{aligned}
& O_{c}=M_{v} \times E_{v}+M_{g} \times E_{g} \\
& M_{v}+M_{g}=I \\
& M_{v} / M_{g}=C_{g} / C_{v}
\end{aligned}
$$

where $O_{C}$ is the output of the fusion module, $\left[\begin{array}{ll}D & \theta\end{array}\right]^{T}$. 
$M_{v}$ is the coefficient matrix of machine vision parameters.

$M_{\mathrm{g}}$ is the coefficient matrix of fusion module 1 parameters.

$E_{v}$ is the matrix of machine vision parameters.

$E_{g}$ is the matrix of fusion module 1 parameters.

$I$ is a identify matrix.

$C_{v}$ is the average error of navigation with only vision sensor.

$C_{g}$ is the average error of navigation with GPS and electronic-compass.

\section{STEERING CONTROL OF THE VEHICLE}

The steering of the vehicle was performed on front-wheel. Therefore the front-wheel angle should be acquired to steer the vehicle. Agricultural vehicles were complicated objects with the parameter variation, environment uncertainty, nonlinear model and so on. However, the fuzzy logic method was presented to decide the front-wheel angle. The steering control algorithm was shown in Fig. 6.

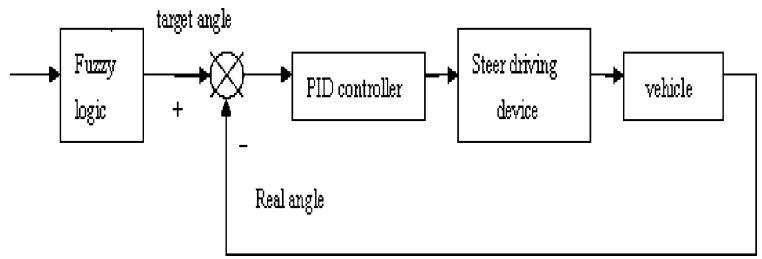

Fig. 6. The steering control algorithm

The input of the fuzzy logic module was the heading angle and offset calculated by the fusion module, the output was the target angle to steer the vehicle. The error between the target angle and the real angle detected by the angle sensor, which was installed on the wheel, was the input of the PID controller. Finally, the steer driving device controlled the vehicle navigation.

\section{CONCLUSION}

An automatic navigation system based on vision sensor, GPS and electronic-compass was developed for agricultural vehicles. The system offered an algorithm to track the directrix between the farm-crop and the non-farm crop. The fusion of the three sensors, vision sensor, GPS and electronic-compass could offer the posture of the vehicle in real-time. The fuzzy logic module was a component to calculate the steering angle and the 
steering controller realized the function of steering with the PID algorithm. The camera was mounted on the cab of the vehicle at operator eye level, and this was proved to be a better algorithm now. The system could navigate the vehicle in a straight line, and through access database the result of different navigation algorithm could be recorded. The redisplaying of the trace could compare the precision of different navigation methods and was used to modify the coefficient of the fusion module to achieve a higher navigation precision. The system could implement the function of automatic navigation in the farmland.

\section{ACKNOWLEDGEMENTS}

This paper is supported by the national 863 projects: Control Technique and Product Development of Intelligent Navigation of Farming Machines (2006AA10A304).

\section{REFERENCES}

Weimin yang, The research on machine vision in agricultural machines, pp.36-48

Qin Zhang and John F. Reid, Noboru Noguchi, Agricultural Vehicle Navigation Using Multiple Guidance Sensors, UILU-ENG-99-7013

Francisco Rovira-Mas, Shufeng Han, Jiantao Wei, John F. Reid, Fuzzy Logic Model for Sensor Fusion of Machine Vision and GPS in Autonomous Navigation, ASAE No.051156

Guangjun Zhang, Machine Vision, Science publishing Company, 2005

Xiaolan He, Research on Algorithms of Localization and Navigation Based on Machine Vision, pp.22-48

Zhigang Zhang, Research of the DGPS Automatic Navigation Control System on Tranplanter, pp.46-59

Zhou Jun, Ji Changying, Liu Chengling, Visual navigation system of agricultural wheeledmobile robot. pp.47-59

Benson, E.R., J.F. Reid, and Q. Zhang. Machine vision-based guidance system for agricultural grain harverters using cut-edge detection .2003BE86 (4), pp.389-398

Benson, E.R., J.F. Reid, and Q. Zhang. Machine vision-based guidance system for an agricultural small-grain harvester 2003 TransASAE46(4), pp.1255-1264

Jiaming Ye, Digital Image Processing and Pattern Reconnition, pp.6-22

Xiaoling Gong, Theory and Simulation Study on Low-Cost Integrated Positioning System of GPS and SINS, pp.35-39

Zhiyan Zhou, Research of multiple sensor combination for agricultural wheel-vehicles navigation pp.13-28 